\title{
Habiter le crassier. Un art anthropocène comme chronique de la catastrophe
}

Living in the Slag Heap: Antropocenic Art as a Way to Chronicle Disasters

\section{Matthieu Duperrex}

\section{(2) OpenEdition}

Journals

Édition électronique

URL : http://journals.openedition.org/transtexts/1075

DOI : $10.4000 /$ transtexts. 1075

ISSN : 2105-2549

Éditeur

Gregory B. Lee

Référence électronique

Matthieu Duperrex, «Habiter le crassier. Un art anthropocène comme chronique de la catastrophe », Transtext(e)s Transcultures 跨文本跨文化 [En ligne], 13 | 2018, mis en ligne le 01 décembre 2018, consulté le 03 juillet 2019. URL : http://journals.openedition.org/transtexts/1075 ; DOI : 10.4000/ transtexts.1075

Ce document a été généré automatiquement le 3 juillet 2019.

(c) Tous droits réservés 


\title{
Habiter le crassier. Un art anthropocène comme chronique de la catastrophe
}

Living in the Slag Heap: Antropocenic Art as a Way to Chronicle Disasters

\author{
Matthieu Duperrex
}

\section{Des formes de vie « exformes »}

1 Selon le commissaire d'art contemporain Nicolas Bourriaud, l'Anthropocène décrit l'élargissement de la « ronde fantomatique » évoquée par Karl Marx au sujet de la formule trinitaire de l'économie, ${ }^{1}$ ce mouvement centrifuge qui excluait le concret (les rapports sociaux de production) pour réifier l'abstrait (la valeur d'échange), qui plaçait à sa périphérie le déchet tout comme l'exploité et rendait les humains comme fantômes d'eux-mêmes. Cette ronde produite par le mouvement industriel aurait entraîné dans son mouvement la planète comme sous-produit du capital. En art, il a souvent été question de refuser les tris induits par le système économique et social, et de ramener à la surface ce que la force centrifuge plaçait à la périphérie :

En art, de Courbet à Gabriel Orozco, un mode de pensée réaliste a nié l'existence du non-assimilable et du déchet, contesté le tri opéré par les appareils idéologiques d'État, promu une vision nominaliste du monde où régnait le singulier, l'exception. Nous voici arrivés à un moment où l'après-coup en vient à dominer le moment historique. Ce qui reste du processus de production, le déchet, a pris une place prépondérante dans la politique, l'économie, la culture. ${ }^{2}$

Ce que Nicolas Bourriaud nomme l'exforme est la procédure de traduction qui émerge à la frontière de l'exclusion et de l'inclusion, du déchet et du produit. L'exforme naît au lieu du partage et de la frontière idéologique, comme étant un geste éminemment politique et esthétique à la fois qui consiste à relever les motifs dépréciés et dévalués de la Modernité. Plutôt qu'une inversion mécanique du centrifuge en centripète, se produit un décentrement, tel celui qui est figuré dans l'Asperge d'Édouard Manet (1880), que ce 
dernier envoie au collectionneur Charles Ephrussi en remerciement de sa générosité, accompagnée du billet suivant : « Il en manquait une à votre botte. » L'enjeu de la forme deviendrait alors d'instaurer des existences moindres par un processus d'intensification : «Sans doute est-ce là une tendance qui traverse tous les arts, la tentative de peupler de nouvelles entités des zones réputées stériles ou inhabitables pour la sensibilité. $»^{3}$

3 Mais il ne suffit pas de replacer le déchet au centre de la création pour faire de l'art. Certes, les métamorphoses du déchet produisent de nouvelles raretés, de la préciosité, de l'art. On songe à la Trousse des naufragés de Jean Arp, aux Merzbau de Kurt Schwitters, à la vaisselle cassée de Raymond Isidore, aux vieux jouets de Bodan Litnianski, à l'art «modeste » d'Hervé Di Rosa et Bernard Belluc... On pense aussi aux Nouveaux Réalistes, aux baraques accumulatives de Ben, aux compressions de César, aux poubelles d'Arman et bien sûr, au Déjeuner sous l'herbe de Daniel Spoerri: un banquet enterré en 1983 et méthodiquement fouillé par l'Institut national de recherches archéologiques préventives (Inrap) en 2010. Et enfin le rebut accède à l'élégance luxueuse avec les drapés en capsules de bouteille d'El Anatsui (fig. 1).

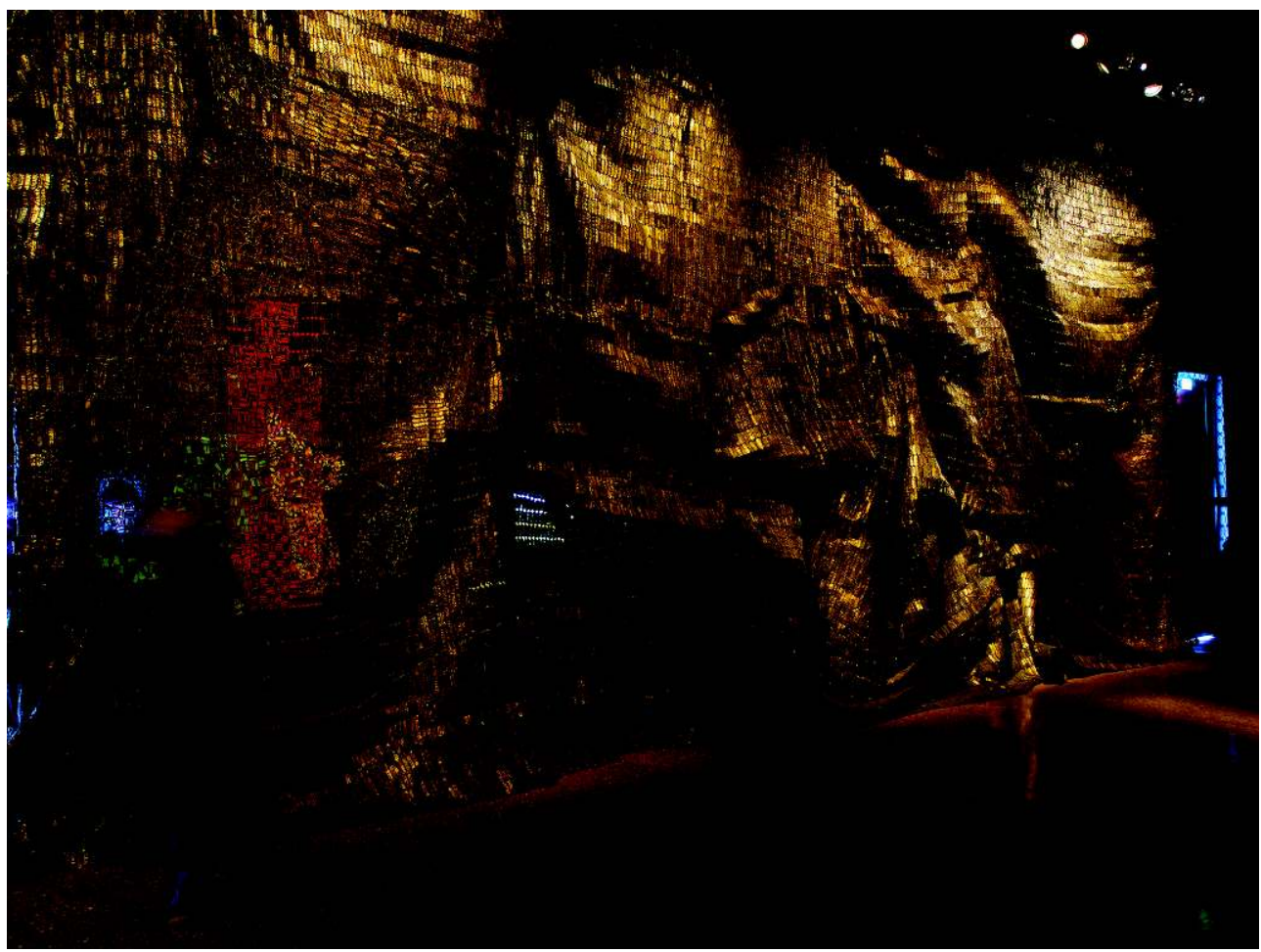

FIg. 1: El Anatsui, The Beginning and the End, 2015 (exposition Intuition, Palazzo Fortuny, Venise, 2017) (C)Matthieu Duperrex

L'enjeu créatif est de «rematérialiser » le déchet plutôt que de parier sur son état d'innocence matérielle en tant que ressource. "Tout est matériel », disait Louis Althusser. Cela signifiait non se positionner au ras du solide et du concret, mais placer les processus de matérialisation au centre de l'attention.

La grande affaire de l'esthétique contemporaine, sa problématique centrale est l'organisation du multiple : les relations prédominent sur les objets, l'arborescence sur les points, le passage sur la présence, le cheminement sur les stations qui le composent. Prises dans des contextes dynamiques, les formes tendent donc naturellement à secréter des récits, à commencer par celui de leur production ellemême, puis celui de leur diffusion: l'œuvre tend ainsi à se présenter comme une 
structure complexe susceptible de générer des formes avant, pendant et après sa réalisation. ${ }^{4}$ graduel à l'existence, le devenir, le tressaillement de «l'instauration précaire $» .{ }^{6}$ Lorsque Thomas Hirschhorn occupe une partie du sous-sol du Palais de Tokyo (Flamme éternelle, 2014), jouant d'un mix énergétique de tas de pneus en labyrinthe, de blocs de polystyrène à sculpter, de cartons et tissus divers, de banquettes et de sièges de voiture défoncés et customisés et d'installations mettant en scène le débordement d'une pensée théorique non assimilable, «ce qui a lieu» est «ce qu'il se passe» et ce qui fait transiter de métamorphose en métamorphose le milieu... happenings permanents modifiant ce paysage fluide de déchets et de matériaux de récupération: "La tâche politique primordiale de l'art contemporain ne consiste pas à dénoncer tel ou tel fait "politique" appartenant à l'actualité, mais à porter la précarité dans les esprits, à maintenir en vie la notion d'intervention sur le monde. $»^{7}$

Dans la performance Touch Sanitation (1979-1980), qui s'étend sur onze mois, Mierle Laderman Ukeles rencontre chacun des 8500 employés du service de la propreté de New York, leur serrant la main et les remerciant personnellement pour leur travail qui «maintient New York en vie ». Ukeles avait publié en 1969 un manifeste féministe et écologiste esquissant un portrait de l'artiste non "comme producteur », mais «comme professionnel de la maintenance ", la troisième partie de ce manifeste consacrant «l'entretien de la Terre ». ${ }^{8}$

7 L'art pourrait alors se définir comme l'instauration et le suivi d'une contamination sensible. C'est d'ailleurs le propos littéral de The House of Contamination du collectif Raumlabor Berlin (2010). Fondée sur le modèle du Fun Palace de Cedric Price, ${ }^{9}$ cette structure créée à Turin à partir d'un matériau de rebuts se voulait un espace public temporaire et un catalyseur d'évènements culturels de tous ordres, avec des interférences ménagées entre les disciplines (d'où la "contamination", qui est à entendre dans le champ artistique des transversalités rendues possibles par le programme architectural). Le paysage de déchetterie agencée se déploie comme composition d'ambiances, comme dans la Nouvelle Babylone de Constant, ${ }^{10}$ à moins que l'ambiance en tant qu'île utopique flottante vienne à visiter elle-même le monde qui l'avait congédiée à la périphérie de ses intérêts, comme lorsque la street-artiste Swoon appareille une flottille de radeaux composés de déchets récupérés dans les poubelles de New York et pilotés par une communauté joyeuse d'artistes se rendant à la Biennale internationale d'art contemporain de Venise, sorte d'embarquement pour Cythère revisité (The Swimming Cities of Serenissima, 2009).

Mais nous aimerions avancer une thèse qui va au-delà de l'analyse rudologique ${ }^{11}$ de créations plastiques à partir de rebuts. Car si nous invoquons ici la terminologie du crassier, ce n'est pas pour parler de la contemporanéité surprenante de la figure du chiffonnier, ${ }^{12}$ ni pour reprendre la spéculation « grotesque » de Parménide et Socrate sur l'eidos de la crasse. C'est plutôt pour prendre en compte ce mouvement d'inversion auquel nous invite l'Anthropocène, ce rapatriement du dehors vers le dedans, de l'environnement « poubellisé » vers les milieux fragilisés, du tiers exclu d'une logique productiviste vers le tiers inclus d'un monde en ruines, et de la prétendue maîtrise de l'ingénierie toute puissante de la technique vers une forme alternative de "souveraineté ", celle qu'évoque Georges Bataille dans un article de 1949, "À propos de récits d'habitants d'Hiroshima », ${ }^{13}$

Transtext(e)s Transcultures 跨文本跨文化, 13 | 2018 
une souveraineté qui ne conduirait pas à une maitrise impérieuse mais qui permettrait de vivre avec la catastrophe.

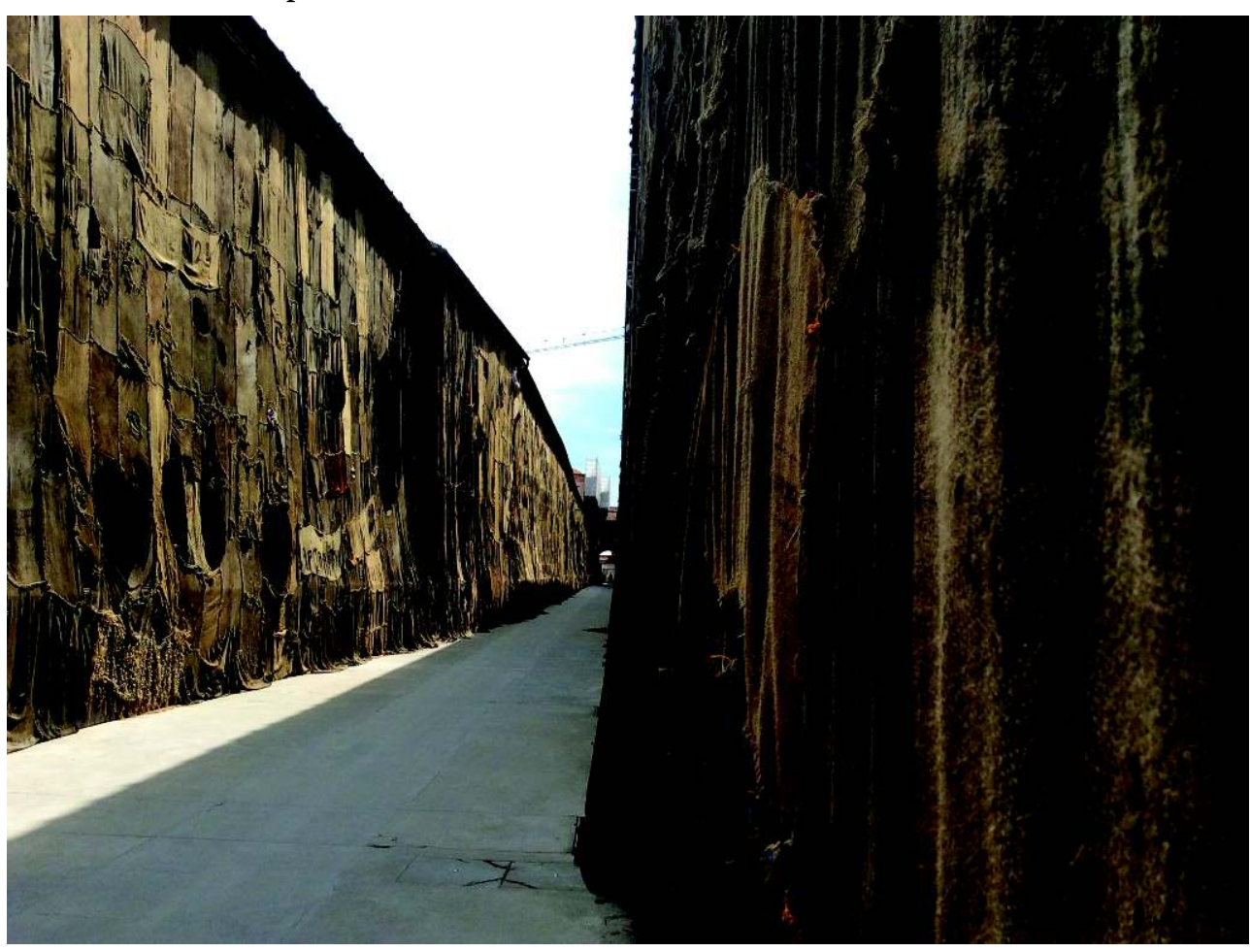

Fıg. 2 : Ibrahim Mahama (1987), Out of Bounds, 2015 (Biennale internationale d'art contemporain de Venise, 2015). Les vieux sacs de jute cousus ensemble portent les noms des différentes denrées (comme le cacao) et de leurs revendeurs, agrégeant les récits de la circulation de la marchandise. Ici, l'artiste utilise essentiellement des sacs qui ont servi au transport du charbon au Ghana. (CMatthieu Duperrex

\section{Sortir de l'économie restreinte}

Bien avant le grand panorama de la civilisation matérielle que dresse le photographe Edward Burtynsky dans Manufactured Landscapes (2003-2006), dont la caméra débute par un long travelling dans une industrie chinoise puis pénètre dans des villages entiers dédiés uniquement au recyclage des déchets électroniques, nous avons en mémoire de nombreuses images de rebut, de tas et de chaos, d'amoncellements détritiques et de résidus d'extraction. De Carleton Watkins (Malakoff Diggings, 1871) aux grands noms de la photographie présentés dans l'exposition New Topographics. Photographs of a Man-Altered Landscape (1975), en passant par Heinrich Zille et son dépotoir illégal (Wilde Müllkippe in Charlottenburg, 1898) et par « Butte, Montana » de Robert Frank (1956), une vue d'un terril de ville minière désolée prise depuis une chambre d'hôtel. Ces images disent l'envers de la production : l'entropie des accomplissements formels d'un monde d'objets - qui sont eux bien achevés, distincts et reconnaissables. Ici, on ne sait pas de quoi il retourne exactement, le crassier est illisible et inhabitable, comme si nous étions tenus à la mauvaise distance d'une expression métamorphique dont le dessein semble absent.

L'anthropologue Anna Tsing a proposé la notion de « diversité contaminée " pour décrire des modes de vie tant biologiques que culturels qui se sont développés à l'occasion des perturbations provoquées ces derniers siècles par l'anthropisation des milieux et leur dégradation..$^{14}$ Avec l'université d'Aarhus, Anna Tsing et ses collègues mènent un 
programme de recherche interdisciplinaire comprenant des artistes et dont l'objet est notamment l'analyse d'une mine de charbon désaffectée au Danemark. ${ }^{15}$ Les observations de ce collectif sur des écologies tourmentées ont alimenté une première expositionrecherche intitulée Mild Apocalypse (Moesgård Museum, 2016) consacrée à cette mine de charbon de Søby comme site expérimental (avec d'autres terrains : un volcan de boue en Indonésie, des projets hydrauliques en Afrique du Sud, des aciéries chinoises, des mines en Espagne, etc.) pour narrer les nouvelles formes de vie qui émergent dans les paysages de l'Anthropocène. Que le terreau en soit un lent dérangement (slow disturbance) ou des altérations profondes et irréversibles, la diversité contaminée serait ainsi l'une des voies à explorer pour considérer le circuit de la valeur depuis des milieux devenus déchets du point de vue de l'économie globale et productiviste jusqu'à une économie locale et holobiotique, comme dans le cas des forêts de pin qui font l'objet de l'enquête sur Le Champignon de la fin du monde. ${ }^{16}$

11 Programme fort, « habiter le crassier » signifie en premier lieu initier le geste artistique depuis l'envers de la production de la Modernité, et cela s'avère impossible sans une démystification préalable de la notion d'économie "globale » du capitalisme dont il faut mesurer le caractère paradoxalement « restreint ». En second lieu, on désigne par cette expression l'établissement d'une fonction résidentielle à l'intérieur des «ruines du capitalisme», au moyen d'une esthétique des attributs d'anti-paysage du sol anthropocénique, et au moyen aussi d'une herméneutique de la traque des allures de la vie qui s'y manifestent.

À l'issue de la seconde Guerre mondiale, Georges Bataille avait établi avec la notion d'économie générale une connexion cosmique ${ }^{17}$ qu'il opposait à l'économie restreinte, ${ }^{18}$ liant les dons inter-corporels à l'exubérance de l'univers et à ses forces intempestives, intimant la pensée éthique et politique à s'ouvrir, de façon constitutive, à une extériorité vaste et turbulente. Décrivant les deux échelles de l'économie restreinte (le régime des fins bornées de l'homo œconomicus, théorisé par les économistes classiques) et de l'économie générale (qui est assez proche de l'acception thermodynamique de Nicholas GeorgescuRoegen) ${ }^{19}$, Bataille mettait en garde sur les dangers qu'il y avait à les séparer :

La méconnaissance par l'homme des données matérielles de sa vie le fait encore errer gravement. L'humanité exploite des données matérielles données, mais si elle en limite l'emploi, comme elle fait, à la résolution (qu'à la hâte elle a dû définir comme un idéal) des difficultés immédiates rencontrées par elle, elle assigne aux forces qu'elle met en œuvre une fin que celles-ci ne peuvent avoir. Au-delà de nos fins immédiates, son œuvre, en effet, poursuit l'accomplissement inutile et infini de l'univers. ${ }^{20}$

13 Si la production capitaliste n'a pas conscience de la pure perte, celle qui arrive de surcroît, des objets qu'elle produit et utilise, c'est qu'elle n'a pas conscience non plus de la valeur énergétique de ces objets, et donc de leur existence telle qu'elle est. Car la production capitaliste a pris l'habitude de ne considérer les existants que lorsqu'ils sont des choses profitables à l'intérêt. Or la pure perte ne peut s'envisager que dans le cadre de l'économie générale, à savoir dans un système fermé où ce qui est perdu l'est irrémédiablement, et n'est pas renvoyé à un ailleurs spatial ou temporel.

Parmi cet excédent et cette dépense incompressible, il y a cet envers de la production que sont les déchets et les pollutions, dont Bataille avait d'ailleurs proposé une forme de science nouvelle, l'hétérologie, ainsi définie :

Science de ce qui est tout autre. Le terme d'agiologie serait peut-être plus précis, mais il faudrait sous-entendre le double sens d'agios (analogue au double sens de 
sacer) aussi bien souillé que saint mais c'est surtout le terme de scatologie qui garde dans les circonstances actuelles (spécialisation du sacré) une valeur expressive incontestable, comme doublet d'un terme abstrait tel qu'hétérologie. (...) Lorsqu'on dit que l'hétérologie envisage scientifiquement les questions de l'hétérogénéité, on ne veut pas dire par là que l'hétérologie est dans le sens habituel d'une telle formule, la science de l'hétérogène. L'hétérogène est même résolument placé hors de la portée de la connaissance scientifique qui par définition n'est applicable qu'aux éléments homogènes... ${ }^{21}$

L'hétérologie est une science oxymorique puisque connaître c'est parvenir à un équilibre statique, c'est s'approprier un objet et le rendre homogène en écartant (expulsion, excrétion) les scories du non essentiel. Les excreta, résultats d'une hétérogénéité, sont ainsi les non-assimilables de la science, de la philosophie et de la religion, même si cette dernière, avec la distinction du sacré et du profane, thématise le rebut plus que tout autre champ de la pensée (hormis la psychanalyse peut-être).

L'Anthropocène met en évidence ce principe de l'économie générale selon lequel les restes qui proviennent de l'activité humaine ne sont pas qu'une forme ultime - compressible ou non- de processus culturels et sous-systèmes productifs d'une civilisation: leur existence est en grande partie solidaire de boucles de rétroaction ${ }^{22}$ biochimiques d'échelle planétaire où l'activité humaine prend place, une place problématique. L'homéostasie de la Terre (selon l'hypothèse Gaia ${ }^{23}$ ) incite à penser un renouvellement du vivant sur fond de réinvestissement des rebuts : il n'y a que très peu de consommation ultime, de nombreux systèmes biologiques répondent à cela, au-delà du cas des populations humaines. On peut se rappeler qu'un scientifique tel que Liebig (1803-1873) a fondé une grande partie du socle de la chimie organique en traquant la «part manquante » d'un optimum de croissance et de rendement: sa mise en évidence des facteurs limitant les rendements agricoles a débouché sur l'invention des fertilisants et intrants chimiques en agriculture. Les azotes et phosphates qui menacent aujourd'hui dangereusement les équilibres biotiques des mers et des océans sont donc directement liés à cette quête du « résidu de croissance ».

17 À la différence du principe de Lavoisier qui anime toujours la chimie moderne, l'hétérologie de Bataille met en évidence ce qui se perd, la perte n'étant pas res nullius, qualification juridique première du déchet, ${ }^{24}$ mais au contraire ce qui pèse dans l'économie générale, appartient à « la matière vivante en général » et au « jeu de l'énergie qu'aucune fin ne limite ». ${ }^{25}$ "La méconnaissance ne change rien à l'issue dernière. Nous pouvons l'ignorer, l'oublier : le sol où nous vivons n'est quoiqu'il en soit qu'un champ de destructions multipliées. ${ }^{26}$ L'hétérologie se présente ainsi comme une intuition métaphysique du régime d'existence dans lequel nous plonge l'Anthropocène. 


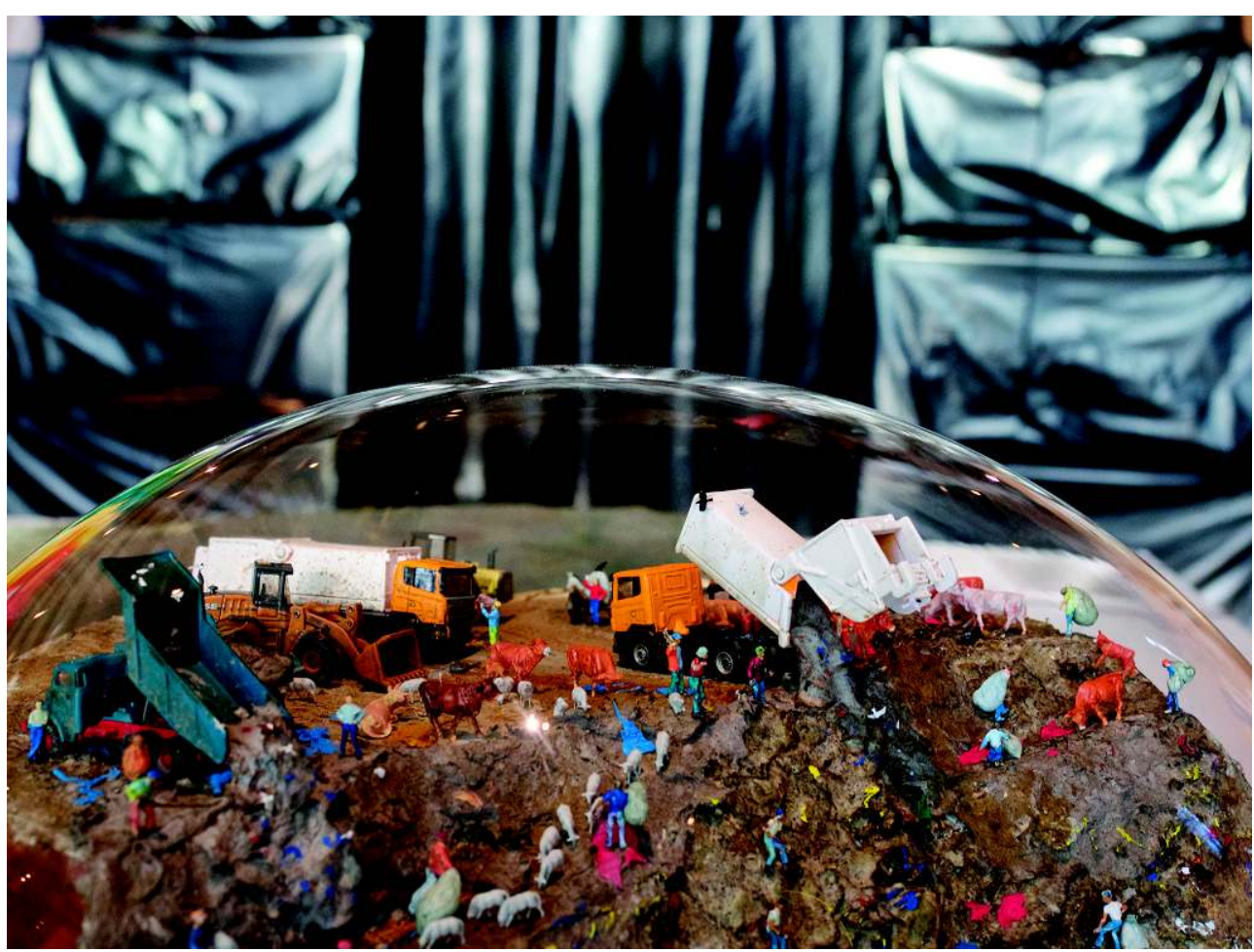

Fig. 3 : Maquette de la décharge de Médiouna, Casablanca (exposition Vies d'ordures, MuCEM, 2017). (c) Matthieu Duperrex

\section{L'étrange souveraineté du malheur}

Écrivant en connaissance de cause depuis l'Anthropocène comme Thanatocène ${ }^{27}$ avec ces « immenses dilapidations » que sont les deux guerres mondiales, Bataille considère, dans toute la puissance de ses effets en termes de " part maudite », la présence « subsidiaire » de l'homme sur la planète :

De même que l'herbivore est, par rapport à la plante, un luxe, - le carnivore par rapport à l'herbivore, - l'homme est de tous les êtres vivants le plus apte à consumer intensément, luxueusement, l'excédent d'énergie que la pression de sa vie propose à des embrasements conformes à l'origine solaire de son mouvement. ${ }^{28}$

Bien sûr, cette grande dilapidation "qui nous anime et même que nous sommes ", ${ }^{29}$ lorsqu'on la saisit, effraie comme un mauvais infini. Mais pour Bataille, l'angoisse est individuelle (c'est-à-dire exprimée du point de vue de l'économie restreinte) et ne peut poindre au point de vue général, non pas par cynisme ou plutôt désinhibition (hypothèse non évaluée mais que nous ferions volontiers nôtre), mais parce que :

En admettant que l'homme est pris dans l'exubérance de la vie, il saisit en même temps que cette exubérance est en lui. C'est sans doute là que se place une manifestation de ce que Bataille nomme la souveraineté, d'une souveraineté qui ne conduit à aucune maîtrise mais qui peut conduire à vivre avec les forces en présence. ${ }^{30}$

Nous retrouvons ici cette notion de souveraineté mentionnée plus haut, lorsqu'écrivant "à propos de récits d'habitants d'Hiroshima", Georges Bataille évoque l'intolérable latitude des souffrances dont ces récits témoignent, comme d'un excès infini à la portée des humains: "Ici ressort l'aspect premier d'une attitude: l'homme de la sensibilité souveraine, regardant le malheur en face, ne dit plus sans attendre "supprimons-le", mais 
d'abord: "vivons-le". (...) En vérité, l'homme est à la mesure du tout possible ou plutôt l'impossible est sa seule mesure. $»^{31}$

Prenons comme hypothèse que le crassier décrit notre habitat contemporain à l'heure de l'Anthropocène, tel que l'hétérologie de Bataille en propose pour la première fois la métaphysique. Que signifie alors vivre et habiter le crassier? C'est sans doute se reconnaître comme sujet de la catastrophe ambiante.

Vivre la catastrophe nous engage à être "consistant" avec la réalité produite par les grands désordres. Cette "consistance" (du latin con-sistere, "tenir ensemble, avoir la même assise") suppose d'une part, l'acceptation que tout individu et toute société sont sujets à la déprise et à la perte de contrôle, qu'une catastrophe demeure quoi que l'on fasse grevée d'injustice, d'absurdité et de laideur, et que nombre d'événements majeurs relèvent $d u$ hors-champ ontologique, épistémique, épistémologique et dramaturgique, bref, qu'une catastrophe est, par définition, le surgissement d'un hors sujet. Mais cette consistance du sujet à l'égard du hors sujet induit, d'autre part, un refus de toute fatalité. Elle engage à un travail du négatif qui, face à la déception, au sentiment d'impuissance et à la déréliction, lutte contre toutes formes d'abandon, de désaffection et de laisser-aller existentiel. ${ }^{32}$

Au-delà de la dramaturgie de la catastrophe nous intéresse la capacité qui est sienne de mettre à nu l'habitabilité du monde et le milieu, l'Umwelt qui en procède. Alors que la catastrophe constitue une rupture d'intelligibilité, un moment de basculement du monde, le champ social travaille à se stabiliser à nouveau au travers d'un collectif, d'un " écosystème » et d'un milieu commun. ${ }^{33}$ Plus que le trauma, est révélatrice la part non événementielle de ce qui est arrivé, l'installation communautaire d'une vie précaire rendue plus silencieuse par la rupture d'une quotidienneté désormais antérieure et la dégradation des sphères techniques et symboliques du milieu habité. Il y a dans ce cas une forme de "latence ontologique " (Merleau-Ponty) où l'individu n'est tout à fait présent ni tout à fait absent. ${ }^{34}$

23 C'est ce travail du commun que saisit par exemple l'architecte Kon Wajirô dans ses dessins. Le tremblement de terre du Kantô survient au Japon le premier septembre 1923. Les villes frappées sont Yokohama, Kanazawa, Shizuoka et Tokyo. Il est 11 h 58 lorsque la terre tremble. Les habitants sont en train de préparer le déjeuner et donc les foyers sont allumés : un immense incendie suit le séisme. On compte cent-cinquante-mille morts. Le nombre de sans-abris est par contre estimé à plus d'un million et demi de personnes. Plus de la moitié des maisons en bois ont disparu et les principales infrastructures ont été détruites. Le milieu habité est donc considérablement affecté. Avant le tremblement de terre, Kon Wajirô a réalisé plusieurs enquêtes dans le Japon rural du nord dans le cadre des études folkloriques (minzokugaku) de l'université de Waseda. Ces études d'architecture vernaculaire trouvent un écho dans la notion de fudô (milieu) telle que la définit le philosophe Watsuji Tetsurô : l'homme est un être relationnel, engagé dans « l'entrelien » ( aidagara) entre le milieu social et l'environnement. Ce couplage est premier. Un aléa catastrophique peut entamer ce couplage par lequel l'individu vit en société et cela, humainement. La catastrophe entraîne une triple « déprise " par laquelle l'individu perd en capacité de prendre (prise technique), de comprendre quoi que ce soit (prise symbolique), de comprendre qui que ce soit (prise sociologique ${ }^{35}$ ).

Lorsque Kon Wajirô décrit l'après-coup du tremblement de terre du Kantô, il propose une ethnographie du relèvement de la Modernité. Selon lui, la catastrophe permet d'observer les conditions primitives de la Modernité et lui donne le "privilège » de voir naître un style tokyoïte influencé par les Américains. À la différence de ce que voudrait la théorie 
des techniques du corps de Marcel Mauss ${ }^{36}$ (et de l'ethnographie japonaise folkloriste ou biologique), le geste n'est pas pour Kon forcément inscrit dans la tradition. C'est le corps tel qu'il se présente immédiatement qui attire son attention, il en étudie toutes les variations avec minutie. C'est ainsi qu'il peut repérer et décrire l'allure des hybrides que sont les modern girls émancipées, alors qu'aucun ethnologue ne s'y est intéressé avant lui. ${ }^{37}$ Le terme kôgengaku est utilisé pour la première fois par Kon Wajirô comme sous-titre de son exposition Shirabemono (" Les objets de l'enquête ») à la librairie Kinokuniya à Tokyo en octobre 1927. Kôgengaku est un néologisme dérivé du terme kôkogaku, l'archéologie. Ko, qui signifie " ancien », est remplacé par la syllabe gen, «le présent ». Kôgengaku est donc un nouveau domaine de recherche, une nouvelle science qui se propose d'utiliser les méthodes de l'archéologie pour étudier le monde présent. C'est «l'archéologie du présent ». Kon Wajirô propose lui-même la traduction littérale du terme kôgengaku en anglais par celui de modernology. ${ }^{38}$

Ces enseignements de la «modernologie » et de l'ethnographie architecturale sont reparus au Japon à l'occasion du tremblement de terre et du tsunami de 2011. Momoyo Kaijima, architecte de l'Atelier Bow-Wow, a ainsi réalisé des enquêtes dans la région côtière d'Ishinomaki-shi, recomposant par le biais de l'interview dessinée le puzzle de la vie qui avait été balayée par le tsunami et réinscrivant le présent dans une continuité temporelle et une " protension » vers le commun futur, grâce au fil de l'enquête. Ainsi, le dessin n'est pas un "plan » au sens d'un projet développé dans un univers de maitrise, il n'est pas sans friction ni négociation et ne présuppose pas de résilience acquise sans que ne se soient au préalable déployés les récits de "souveraineté ${ }^{39}$ Nous voyons ainsi comment habiter le crassier prend consistance pour des sujets d'un drame et d'un destin commun. Dès lors, comment l'art contemporain, et non nécessairement un prétendu « art écologique $",{ }^{40}$ se saisit-il de ce contexte oppressant et de basculement du monde dans la catastrophe que nous résumons dans la formule "habiter le crassier»? Comment traduit-il au moyen des voies esthétiques la situation de précarité qui est la nôtre en Anthropocène et dont les sciences humaines prennent aujourd'hui argument pour redéfinir la condition première de l'humain, habiter? Nous proposons ci-dessous sept exemples commentés nous permettant d'élaborer une réponse.

\section{Chroniques de la diversité contaminée}

Basé à Tokyo, le collectif d'artistes Chim $\uparrow$ Pom réalise performances et installations à un rythme soutenu..$^{41}$ L'humour et la satire font souvent partie de leur répertoire critique de la société japonaise. Dans l'installation vidéo Black of Death (2013) on suit l'une des membres du collectif qui attire à elle de nombreux corbeaux grâce à un leurre et à un mégaphone qui diffuse un enregistrement de croassement. Ces actions qui mêlent spontanéité et étrangeté prennent place dans des lieux symboliques de Tokyo, tels que Shibuya ou la place du Parlement. Les corbeaux sont très nombreux au Japon où ils jouent volontiers le rôle d'éboueurs indésirables. La Ville de Tokyo reçoit en moyenne six-cent plaintes par an d'habitants ayant été attaqués par des corbeaux. Il se trouve en effet qu'ils sont assez territoriaux et agressifs, surtout au printemps. Les corbeaux ont notamment proliféré aussi dans l'ancienne zone d'évacuation de Fukushima pour l'essentiel toujours déserte bien qu'accessible. Le montage vidéo met en parallèle ces oiseaux filmés dans les ruines de Fukushima et la performance de ralliement des corbeaux à Tokyo à l'aide d'un oiseau empaillé et d'un cri de détresse. Bientôt, des centaines d'oiseaux suivent le 
véhicule - voiture ou scooter - de la jeune-fille hilare. On sent l'étonnement teinté d'inquiétude des passants qui assistent à la scène. La situation est très différente de la scène d'anthologie d'Hitchcock, l'angoisse est d'une tout autre nature. De quoi les corbeaux sont-ils les émissaires ? Leur vacarme annonce-t-il une nouvelle catastrophe ? Le lien que Chim $\uparrow$ Pom ménage avec la zone contaminée de Fukushima sonne comme un rappel bruyant et indélicat. Là-bas la contamination est silencieuse, elle appartient aux mondes infimes des radionucléides que seuls les compteurs Geiger révèlent ; l'appareil de mesure y est le seul révélateur de la "matière vibrante » qui peut nous nuire. ${ }^{42}$ L'effet sonore des corbeaux est distinct, il ne relève pas de la mesure d'un danger sournois mais du soulèvement sauvage d'un environnement sous pression. Ce débordement de la nature liminaire, rendue à l'autonomie, cette irruption cacophonique est « dérangeante » au sens littéral, car elle grève d'inutilité toute stratégie d'évitement ou bien d'approche euphémisante de la catastrophe par un retour à l'ordre de façade. Le croassement d'alerte de ces corbeaux sentinelles brise un silence, une apparente santé dont Bichat disait que c'était « la vie dans le silence des organes». Ces corbeaux sont gaïesques. Comme le dit Isabelle Stengers, ${ }^{43}$ nommer Gaia, c'est le premier acte de composition avec ce qui arrive. On ne lutte pas contre Gaia, cela n'a aucune signification. La prise de signification, et la possibilité d'action commune et de pensée, la première prise donc pour composer le commun, c'est cela : nommer ce qui nous arrive... ou bien croasser dans le crassier.

The Ferryman ("Le Passeur des lieux », 2016) semble proposer un déploiement depuis cette instance de désignation de la catastrophe, et cela au moyen d'un schème de la réparation qui passe par le rite initiatique. ${ }^{44}$ Le Passeur des Lieux est un film de Gilles Delmas, avec les chorégraphies de Damien Jalet, la participation de la performeuse Marina Abramović (voix-off) et du compositeur Ryuichi Sakamoto. Il se présente comme une exploration cinématographique et chorégraphique des racines animistes des rituels, de la danse et de la sculpture. Scénographiée dans les paysages des îles volcaniques comme Bali, le Japon et l'Ecosse, ainsi que dans le musée du Louvre et l'Opéra de Paris, la relation ancestrale et complexe des hommes et de la nature, souvent considérée comme une passerelle entre les mondes visibles et invisibles, est représentée ici par une série de cérémonies frappantes - transe, crémations et sacrifices rituels à Bali, culte de la montagne au Japon -, des spectacles de danse contemporaine, des extraits de textes et une parabole, narrée par Marina Abramović. Grâce au parcours d'un homme mi-cerf, michasseur, interprété par le danseur Damien Jalet, ce film déroule la métaphore poétique et viscérale de l'ascension en six étapes d'une montagne, considérée comme lieu sacré de naissance et de mort. Ces six étapes sont des illustrations du «sauvage» non comme catégorie de classement, par opposition au "domestique», mais comme une forme d'expression intime de l'individu, qui a pour corollaire la liberté créative. Le film met en scène plusieurs intercesseurs qui permettent à l'homme-cerf d'accomplir son cheminement. Ce sont des moines, des chamans. Une fois sorti de la matrice et du chaos (première étape), le personnage est confronté aux arrières-mondes des esprits (deuxième étape) puis au conflit intérieur entre animalité et humanité (troisième étape). Le personnage s'affirme comme guerrier, combattant vaincu, devant retourner au sol (quatrième étape). Il se confronte ensuite à la violence de l'arrachement à la nature par la puissance de destruction des hommes (cinquième étape, se déroulant à Fukushima). La sixième étape, celle de l'arrivée au sommet de la montagne est une réflexion sur la nature transformée en ressource, les sacrifices devenant d'échelle mondiale. L'ensemble de ce parcours est complexe, ponctué de beaucoup de pièces chorégraphiques et de rites 
relatifs à diverses croyances. Seule la voix-off de Marina Abramović assure une continuité de propos. Bien que mystique, la forme d'ensemble constitue l'exposé d'une sorte de propédeutique pour connaître les équilibres du monde, la partition entre visible et invisible, l'enchevêtrement des différents êtres qui composent la nature au-delà des catégories dualistes sauvage/domestique, naturel/artificiel, nature/culture. On remémore ce qui a peuplé le monde et qui a été effacé, et comment l'on peut repeupler à partir d'une révélation du sauvage ce monde rendu nôtre. Cette capacité à honorer, préserver, régénérer le sauvage dans les ruines est sans doute l'un des paradigmes de la création artistique qui nous permet d'habiter le crassier.

La prolifération des crassiers est quelque chose de très commun dans l'Anthropocène. C'est pourquoi leur mise en réseau s'impose souvent comme stratégie d'enquête. D'où la création de "plateformes pour de nouvelles écologies ", selon l'expression d'Emily Eliza Scott qui se demande à quelle « nouvelle espèce institutionnelle » on a ainsi affaire et qui compare les artistes enquêteurs à des « coyotes " plein de ressources, sachant s'adapter aux conditions mouvantes des nouveaux territoires. ${ }^{45}$ World of Matter ${ }^{46}$ réunit ainsi plusieurs artistes, architectes et documentaristes autour d'un média international diffusant des enquêtes artistiques sur les ressources écologiques. ${ }^{47}$ Outre les documentations en ligne de la plateforme, très soignée, le collectif coordonne symposiums, publications et expositions; il est en partie commissariat artistique, en partie organisme de recherche tissant des liens avec la communauté scientifique.

En mettant en relation un document visuel sur l'exploitation aurifère illicite dans le bassin amazonien avec un fichier vidéo sur les états pétroliers du delta nigérian ou sur les politiques égyptiennes de gestion des sols, nous suggérons une variété de lectures possibles sur la connectivité mondiale entre ces sites. Plus important encore, nous nous intéressons au potentiel créatif des écarts entre différents sites une fois que les cadres conventionnels sont levés, et aux nouveaux paysages de flux de ressources qui pourraient ainsi émerger. ${ }^{48}$

World of Matter revendique des méthodologies diverses et présente ainsi une plasticité des approches narratives qui permet un regard renouvelé sur la matérialité de la Modernité et sur ses pratiques discursives :

Sa créolisation des angles disciplinaires pour les repenser dans un rapport complexifié avec le réel résiste à toute prétention d'articuler notre être-au-monde en dehors des contingences matérielles, l'existence humaine étant pour ce collectif nécessairement "formée, déformée et transformée" par les objets et les matières. ${ }^{49}$

Dans la copieuse production de World of Matter, on peut notamment distinguer l'enquête Traces of Nitrate de Xavier Ribas, ${ }^{50}$ qui mène depuis 2009 une archéologie fouillée des mines chiliennes de nitrate de sodium, exploitées entre 1870 et la fin des années 1920, essentiellement par les Britanniques. Ribas trace les circuits de transformation du minéral en capital sur les places boursières londoniennes et offre une approche socioculturelle d'une industrie ambivalente, qui débouche sur les fertilisants comme sur les explosifs, sur la vie et sur la mort. Dans ses expositions, comme celle produite au MACBA en 2014, les relevés paysagers photographiques ou vidéo du désert d'Atacama croisent les documents d'archive (ainsi que la mise en scène de la recherche) comme la collection bigarrée des paquets de cigarettes que fumaient les mineurs.

31 Deep Weather d'Ursula Biemann (2013), elle aussi membre du collectif World of Matter, est pour sa part un essai vidéo qui se distingue du strict genre documentaire par un parti pris de mise en relation, selon un "effet papillon ", entre les sables bitumineux d'Alberta, au Canada, et le delta sud du Bangladesh. D'un côté, les « géologies du carbone », de l'autre, 
les « hydrographies ». L'artiste témoigne en voix-off de la « terrible beauté » des paysages ravagés de la forêt boréale, ou bien promis à la submersion du fait de la fonte accélérée des neiges himalayennes et de la montée du niveau de la mer. Le pétrole et l'eau sont ainsi mis en relation dans une logique causale qui dénonce la part des industries du carbone dans le réchauffement climatique. Mais Ursula Biemann ne cherche pas à réduire la complexité "des géographies mouvantes et des temporalités divergentes ", ${ }^{51}$ elle montre plutôt comment elles se contaminent l'une l'autre. Ce n'est pas tant qu'elle propose une simplification ou un raccourci qu'elle pointe un déséquilibre anthropologique, réalisant au Bangladesh de longs portraits cadrés et filmant à hauteur d'homme une procession d'hommes et de femmes construisant de leurs mains une digue dérisoire destinée à protéger leur village et leurs cultures des crues. À ce désarroi de la lutte pour la survie s'oppose le "sublime toxique" auquel le photographe Edward Burtynsky nous a accoutumés, ${ }^{52}$ d'immenses champs bitumineux filmés de haut, dépouillés de présence humaine et où l'on ne distingue que d'énormes engins de chantier. Pour Ursula Biemann, les «ur-liquides » que sont le pétrole et l'eau sont des puissances sous-jacentes aux narrations des changements qui affectent l'écologie planétaire, ils ont une agentivité et sont considérés comme des acteurs de la dramaturgie. Le préfixe " ur » dénote en Allemand l'originaire, l'arché en Grec ancien, une impulsion sourde et profonde ou une animation primordiale qui perdure dans le mouvement des êtres. Ainsi, c'est une forme d'antagonisme chthonien qui s'exprime et exige des communautés affectées, les natifs amérindiens comme les paysans bangladeshi, un sacrifice infini, une dépense somptuaire où s'abîme leur futur.

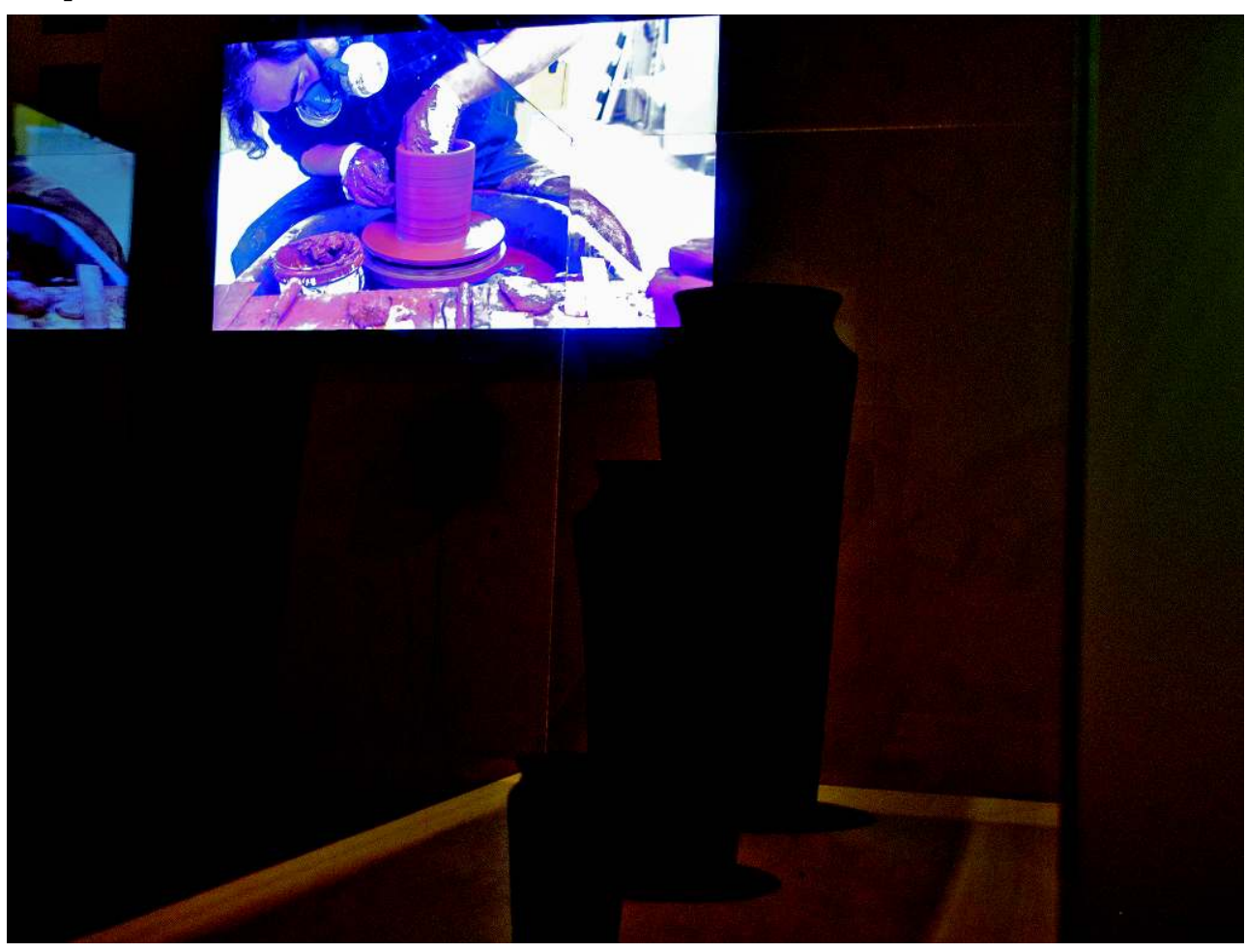

Fig. 4: Unknown Fields Division, Rare Earthenware, 2015 (exposition Reset Modernity!, ZKM, 2016). CMatthieu Duperrex

Outre la contamination narrative qui procède de démarches d'enquêtes empiriques, on peut aussi relever l'intégration critique du potentiel métamorphique du crassier dans les chaînes opératoires artistiques. C'est le cas avec le projet Rare Earthenware (2015, fig. 4). 
Le collectif Unknown Fields Division, ${ }^{53}$ en collaboration avec le céramiste Kevin Callaghan, a ainsi fabriqué trois céramiques radioactives pour une exposition du Victoria and Albert Museum intitulée "What is Luxury» (25 avril-27 septembre 2015). Les vases arborent une forme classique en céramique chinoise, mais ils ne sont pas peints. Ils sont en grès noir et en résidus miniers. ${ }^{54}$ De tailles différentes, ces trois vases sont dimensionnés en proportion exacte à la quantité de déchets toxiques générés par la fabrication de trois objets manufacturés high-tech : le smartphone, l'ordinateur portable et la batterie « intelligente » d'une voiture électrique. Ainsi que le montre le documentaire vidéo qui accompagne l'exposition des vases, le matériau a été collecté dans un lac radioactif de Mongolie intérieure, à Baotou, où sont rejetés les déchets de raffinage des terres rares, les dix-sept matériaux recherchés pour produire de l'électronique haut de gamme. Les vases sont décrits par leurs concepteurs comme étant un témoignage de "l'ombre toxique que nos objets de tous les jours projettent sur la terre ».

Comment l'enquête artistique détache-t-elle de l'arrière-plan spatio-temporel du récit un événement, biographique ou historique, qui serve d'épisode clé à la description de la situation? Comme dit John Dewey, « un événement est strictement ce qui sort ; ce qui fait une sortie $\aleph^{55}$; Dewey emploie le terme eventuation, ampliation du verbe eventuate qu'on peut rendre par "parachèvement ». Le vocabulaire géologique nous permet d'illustrer cette indexation temporelle. Les impasses et les fantômes de l'histoire qui reviennent nous hanter motivent les récipiendaires franco-libanais du Prix Marcel Duchamp 2017.56 Joana Hadjithomas et Khalil Joreige sont des artistes réalisateurs qui aiment révéler des histoires secrètes. Le projet plastique plurimédia, Discordances (fig. 5), s'intéresse aux histoires en strates qui sont dans le sol des villes, à Paris, Beyrouth et Athènes. Deux unités temporelles se retrouvent en collision sous l'effet d'une catastrophe ou d'un glissement de terrain, puis le sol se stabilise au-dessus et se régénère: c'est cela en 
géologie, les discordances (unconformities). Les pierres gardent une mémoire de certains faits et racontent aussi les stratégies d'occupation humaine de la terre.

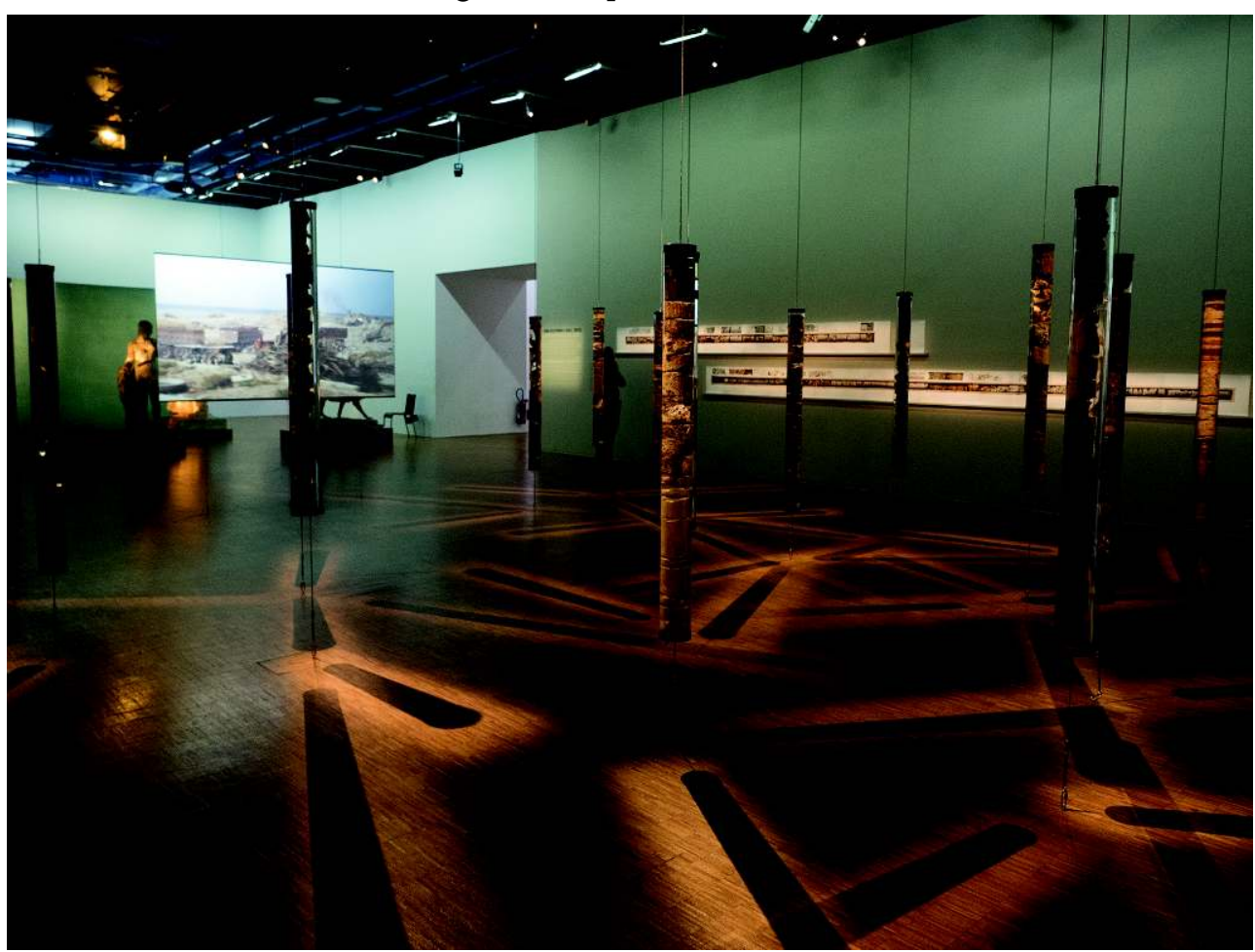

Fig. 5 : Joana Hadjithomas et Khalil Joreige, Discordances, 2017 (Prix Marcel Duchamp 2017, Centre Pompidou). (Matthieu Duperrex

Nouant une collaboration avec des scientifiques dans le cadre d'opérations d'archéologie préventive, ils ont extrait de grandes carottes " messagères du sous-sol ", ${ }^{57}$ time capsules naturalisées d'une histoire humaine rendue à l'état géologique. En ce sens, le projet de Joana Hadjithomas et Khalil Joreige, bien que proche du point de vue plastique, est très différent de celui d'Alan Sonfist. La composition sculpturale et minimaliste des Earth Monuments d'Alan Sonfist (Earth Monument to Chicago, 1965-1977 ; Earth monument to New York, 1979) reposait en effet sur une succession de carottes de forage classées selon un ordre chromatique du plus clair au plus foncé (sable, argile, limon gris, gravier, schiste vert, grès, calcaire). Comme dans le cas du sanctuaire végétal Time Landscape (1968), revenant à un temps antérieur à la colonisation de Manhattan, Sonfist ne prélève que des couches de sol «naturelles" pour les « rétorquer » aux civilisations urbaines modernes. Tout autre est le projet archéologique des Discordances. Paradoxalement, c'est parce que le sol est voué à disparaître (du fait de la croissance urbaine et de la spéculation immobilière) que des histoires vont pouvoir en être exhumées. L'archéologue détruit pour préserver une mémoire, et il doit aussi parfois laisser en jachère un sol pour que ses successeurs puissent travailler sur ce patrimoine. Cette idée de laisser volontairement des découvertes potentielles dans la terre pour les temps futurs questionne bien évidemment la relation inverse du capitalisme extractif aux ressources du sol.

La discontinuité ou discordance peut être considérée comme une notion géologique et comme une propriété de la matière historique. Alors que ces feuilletés rangés dans des boîtes se décomposent généralement très vite, Hadjithomas et Joreige ont cherché avec l'artiste Adam Lowe et son studio de production Factum Arte une solution pour mettre en valeur ces singuliers nouveaux « élevages de poussière » à l'intérieur de grands cylindres 
de verre remplis d'une résine transparente spéciale. Par endroit dans les tubes, on ne sait pourquoi, une réaction d'éclatement de la résine s'est produite, une brisure intérieure qui est une nouvelle discordance, à l'intérieur de la chaîne opératoire artistique cette fois-ci. $\mathrm{Au}$ mur, les frises chronologiques (les logs qui sont comme les rushs d'un film) sont reconstituées, impeccablement dessinées et l'enchaînement des discordances précisément relaté au travers des annotations des archéologues sur la stratigraphie.

L'enquête parvient ici à converser avec les fantômes de nos milieux, et parfois à en faire des colocataires de notre habitat. Habiter le crassier c'est pouvoir s'associer comme coexistants ces fantômes ou ces monstres de l'Anthropocène, pouvoir capter leur parachèvement dans le cours spatio-temporel de l'enquête. "Ce qui existe co-existe, et aucun changement ne peut soit se produire soit être déterminé dans l'enquête, isolé de la connexion d'une existence avec les conditions co-existantes. $1^{58}$

La Modernité a inventé un ensemble de dispositifs ou de postures commodes de pensées qui permettent de faire fi de ces connexions en instituant des partages catégoriques, puis en bricolant une succession de passe-droits pour sauter pardessus l'abîme (la fameuse doctrine saltatoire de la vérité décrite par William James ${ }^{59}$ ). Guère étonnant alors que les fantômes et les monstres dansent pour les Modernes une "ronde fantomatique » qui les tétanisent et les rendent impuissants face aux boucles de rétroaction de Gaia! Mais à leur décharge, s'attacher à des fantômes est ingrat et peut rendre l'existence très concrètement "impossible ». Les altérations de l'air et du sol produisent certains monstres avec lesquels on ne peut pactiser, avec lesquels l'on ne peut qu'entrer en guerre...

Dernier exemple que nous convoquerons, Leviathan, film de Lucien Castaing-Taylor et Véréna Paravel (2012), inaugure un genre nouveau, la « sensory ethnography ». Issu de deux ans de recherche en haute mer avec des pêcheurs de Nouvelle-Angleterre, il invite à une plongée dans l'océan au travers de la pêche industrielle. Mais les portraits que dresse le film ont pour singularité de résister tant à l'idéalisation romantique du dur labeur des travailleurs de la mer qu'à l'anthropocentrisme : le bateau, les mouettes, les poissons, les coquillages bénéficient d'une mise en scène identique et tous ensemble participent d'une cosmologie sans grand récit mythique.

Il n'était pas question de leur faire croire qu'ils étaient les réalisateurs mais de faire intervenir leurs corps, leur vécu de manière plus ou moins consciente pour obtenir un film qui nous plonge avec les hommes au cœur des éléments, comme si la mer, les oiseaux, les pêcheurs écrivaient le film avec nous. ${ }^{60}$

Comme Melville dans Moby Dick, nous partons du port de New Bedford, ancienne capitale mondiale de la chasse à la baleine. L'embarquement est aussitôt ressenti physiologiquement, par le choix technologique de caméras accrochées un peu partout sur le chalutier, y compris dans les filets, ou suspendues au bout de mats pour accompagner au plus près le mouvement des oiseaux suivant le sillage. Ce qui pourrait être conçu comme un contrôle panoptique esthétisant est au contraire une manière de suivre la prolifération des existants jusque dans leur chair, la scène de pêche étant une grande symphonie orchestrale qui mêle tous les matériaux de la perception jusqu'à la confusion des sens. Leviathan, à la fois métaphore politique de la souveraineté et monstre biblique cataclysmique, est une somptueuse et cruelle leçon d'anthropologie politique de la grande dilapidation " qui nous anime et même que nous sommes ", comme écrivait Bataille.

Enquête ethnographique, innovation technique, recherche formelle, poème cosmique, aventure contemporaine lestée d'un vaste héritage cinématographique, littéraire et mythologique: en combinant ces aspects hétérogènes dans une 
expérience perceptible, Leviathan produit un impact impressionnant sur le film d'essai. ${ }^{61}$ refuge mais au contraire une expression de la souveraineté dans les ruines. Nous sommes parties prenantes d'une odyssée de fin du monde, où la vie et la mort, le machinique et l'organique, les moyens de production et la marchandise peuvent tournoyer d'une « ronde fantomatique » dans l'œil d'un poisson agonisant.

\section{NOTES}

1. «Dans la formule capital-profit, ou, mieux, capital-intérêt, terre-rente foncière, travail-salaire, dans cette trinité économique qui veut établir la connexion interne ente les éléments de valeur et de richesse et leurs sources, la mystification du mode capitaliste de production, la réification des rapports sociaux, l'imbrication immédiate des rapports de production matériels avec leur détermination historico-sociale se trouvent accomplies ; et c'est le monde enchanté et inversé, le monde à l'envers où monsieur le Capital et madame la Terre à la fois caractères sociaux, mais en même temps simples choses, dansent leur ronde fantomatique. " (Karl Marx, Le Capital, livre III, tome III, chap. 48, Paris, Éditions sociales, 1974, pp. 207-208)

2. Nicolas Bourriaud, L'exforme. Art, idéologie et rejet, Paris, Presses universitaires de France, coll. «Perspectives critiques », 2017, pp. 139-140.

3. David Lapoujade, Les Existences moindres, Paris, Éditions de Minuit, coll. «Paradoxe », 2017, p. 86.

4. Bourriaud, L'exforme, p. 79.

5. Cf. Platon, Parménide, $103 \mathrm{sq}$ : «Il n'est pas au pouvoir du non-être d'empêcher l'être de se constituer tout entier; il n'est pas au pouvoir de l'être, de faire qu'il y ait ici ou là plus ou moins d'existence. »

6. Étienne Souriau, Les différents Modes d'existence, Paris, Presses universitaires de France, coll. «Métaphysiques », 2009, p. 161.

7. Bourriaud, L'exforme, pp. 74-75.

8. Maintenance Art Manifesto 1969! Proposal for an Exhibition "CARE" (1969).

9. D'une part, le Fun Palace est inspiré par la philosophie égalitaire des terrains d'attractions anglais du XVIII ${ }^{\mathrm{e}}$ siècle, comme Vauxhall et Ranelagh, avec leurs immenses espaces pour la promenade, le jeu et les commérages. D'autre part, le projet non réalisé de Price est actuel, se fait l'interprète des théories novatrices de cybernétique, des principes du théâtre d'avant-garde, des 
technologies de pointe et d'un humour libre, à la Monty Python. L'objectif ultime est de créer un bâtiment modifiable en fonction des désirs des utilisateurs.

10. Dans la New Babylon dont Constant Nieuwenhuys construit la maquette dès 1958, «l'imagination des habitants dessine la ville en créant une multitude d'espaces aux ambiances propres. Tous les habitants sont libres d'y contribuer et d'apporter leur pierre à une œuvre éphémère.»

11. La rudologie (du latin rudus, décombres) désigne l'analyse raisonnée et scientifique des déchets en tant que témoins de sociétés données et de leurs inscriptions spatiales. On doit ce terme en France à Jean Gouhier (cf. sa thèse, Éléments pour une Géographie des déchets, 1972). L'Américain William Rathje en a fait une dimension à part entière de l'archéologie.

12. Cf. Baptiste Monsaingeon, Homo detritus. Critique de la société du déchet, Paris, Éditions du Seuil, coll. « Anthropocène ", 2017.

13. Georges Bataille, CEuvres complètes, XI, Articles 1, 1944-1949, Paris, Gallimard, 1988, p. 185.

14. Cf. Anna Lowenhaupt Tsing, "Contaminated Diversity in "Slow Disturbance": Potential Collaborators for a Liveable Earth », RCC Perspectives, ${ }^{\circ}$ 9, 2012, pp. 95-98.

15. Cf. Anna Lowenhaupt Tsing, "Unintentional design in the Anthropocene ", in AURA Working Papers, vol. 1, « More than human », Aarhus University, 2015, www.anthropocene.au.dk

16. Anna Lowenhaupt Tsing, Le Champignon de la fin du monde. Sur la possibilité de vivre dans les ruines du capitalisme, traduit par Philippe Pignarre, Paris, La Découverte, coll. " Les Empêcheurs de penser en rond ", 2017.

17. Cf. Cédric Mong-Hy, Bataille cosmique. Georges Bataille, du système de la nature à la nature de la culture, Paris, Lignes, coll. « Fins de la philosophie », 2012.

18. Georges Bataille, La Part maudite, Paris, Éditions de Minuit, coll. « Critique », 1980 [1949].

19. $\mathrm{Cf}$. Jacques Grinevald, «La révolution industrielle à l'échelle de l'histoire humaine de la biosphère ", Revue européenne des sciences sociales, nXLIV-134, 2006, pp. 139-167.

20. Georges Bataille, La Part maudite, p. 59.

21. Georges Bataille, Euvres complètes, II, Écrits posthumes, «La valeur d'usage de D.A.F. de Sade », Paris, Gallimard, 2002, pp. 61-62.

22. Les phénomènes qui entrent sous la catégorisation du réchauffement climatique ne sont pas unitaires. Ils sont désignés par les scientifiques au travers d'un ensemble de rétroactions des cycles bio-géo-chimiques planétaires en réponse à l'activité anthropique. Afin qu'il n'y ait pas d'ambiguïté, nous utilisons ici l'appellation de Gaia pour rassembler un complexe hétérogène et hétérarchique de boucles de rétroaction. Gaia n'est certainement pas un super organisme, elle est plurielle. Pour une mise au point, voir Bruno Latour et Timothy M. Lenton, «Extending the Domain of Freedom, or Why Gaia Is So Hard to Understand », Critical Inquiry vol. 45, n³, 2019, pp. 659-680. https://doi.org/10.1086/702611.

23. Cf. James E. Lovelock et Lynn Margulis, « Atmospheric homeostasis by and for the biosphere: the gaia hypothesis ", Tellus $6, \mathrm{n}^{\circ} 1,1974, \mathrm{pp} .2-10$.

24. Tant à l'échelle locale que dans la "mondialisation discrète », selon la belle expression du géographe Olivier Pliez, nous savons pertinemment que ce que nous jetons n'appartient peutêtre plus à personne, comme le dit le droit (le déchet est ce qui «tombe » du bien), mais entre volontiers à nouveau dans l'engrenage de l'économie marchande. De la réduction à la source à la réinvention des modes d'existence de la matière rebut, des enjeux économiques sont présents, et sans doute pas si récents que cela. Du point de vue des normes et de l'appareillage juridique, du point de vue des formats sociaux et institutionnels qui entourent cet aspect, il y a matière notamment à interroger la notion de «bien». Comment se valorise et prend consistance ce «bien commun » qu'est le déchet, ce waste common (l'expression est de Ruth Lane à propos des chiffonniers australiens) ? L'exposition du MuCEM en 2017, Vies d'ordures, essayait de formuler une réponse contemporaine avec les moyens de l'enquête anthropologique. Cf. Denis Chevallier 
et Yann-Philippe Tastevin (dir.), Vies d'ordures. De l'économie des déchets, Marseille, Éditions Artlys, 2017.

25. Georges Bataille, La Part maudite, op. cit., p. 61.

26. Ibid., p. 62.

27. «L'efficacité a un sens très différent lorsque l'enjeu est de tuer plutôt que d'être tué, écrivent les historiens Bonneuil et Fressoz. L'évolution des systèmes d'armement contemporains illustre cette tendance à l'exubérance énergétique, intrinsèque au fait militaire. Pendant la Seconde Guerre mondiale, la troisième armée du général Patton consommait 1 gallon de pétrole $(3,7$ litres) par homme et par jour. On atteint 9 gallons pendant la guerre du Vietnam. 10 pour l'opération "Tempête du désert" et 15 durant la seconde guerre du Golfe. Les technologies militaires actuelles atteignent des degrés inégalés de consommation énergétique. » (Christophe Bonneuil et Jean-Baptiste Fressoz, L'événement Anthropocène. La Terre, l'histoire et nous, Paris, Éditions du Seuil, coll. « Anthropocène », 2013, pp. 144-145)

28. Georges Bataille, La Part maudite, pp. 75-76.

29. Ibid., p. 77.

30. François Dutrait, « L'impossible à exposer ", L'en-je lacanien, $n^{\circ} 25,2015$, p. 46.

31. Georges Bataille, CEuvres complètes, XI, Articles 1, 1944-1949, p. 185.

32. Yoann Moreau, « Des catastrophes « hors sujet » ", Communications, n96, 2015, p. 17.

33. Cf. Yoann Moreau, Vivre avec les Catastrophes, Paris, Presses universitaires de France, coll. «L'écologie en questions », 2017, p. 92.

34. Ibid., p. 117.

35. Ibid., p. 146.

36. Cf. Marcel Mauss, «Les techniques du corps» (1935), in Sociologie et anthropologie, Paris, Presses universitaires de France, coll. « Bibliothèque de sociologie contemporaine », 1950.

37. Cf. Damien Kunik, "Gestes et "archéologie du présent" au Japon des années 1920 à aujourd'hui. Quelques enseignements pour l'ethnologie francophone », Techniques \& Culture, $\mathrm{n}^{\circ} 62$, 2014, pp. 68-83.

38. Cf. Harry D. Harootunian, Overcome by Modernity. History, Culture, and Community in Interwar Japan, Princeton, Princeton University Press, 2000.

39. Cf. Momoyo Kaijima, Laurent Stalder et Yu Iseki (dir.), Architectural Ethnography, Tokyo, TOTO, 2018.

40. Bénédicte Ramade a montré dans plusieurs articles que l'art écologique peine à mobiliser sur ses objets selon une doctrine de «l'éveil des consciences » assez médiocre, ou bien a du mal à démontrer des qualités esthétiques qui métamorphosent réellement le regard du public, qui l'installent «dans le trouble». Cf. Bénédicte Ramade, "L'art de l'écologie aux limites de l'exposition », Espace: Art actuel, n¹10, 2015, pp. 12-21 ; Bénédicte Ramade, «L'art écologique aux prises avec ses stéréotypes ", Perspective, $n^{\circ} 1,2015$, pp. 184-90.

41. Consulter le site Internet de Chim $\uparrow$ Pom : http://chimpom.jp

42. Cf. Sophie Houdart, "Les répertoires subtils d'un terrain contaminé », Techniques \& Culture, vol. $68, \mathrm{n}^{\circ} 2,2017$, pp. 88-103.

43. Cf. Isabelle Stengers, Au temps des catastrophes. Résister à la barbarie qui vient, Paris, La Découverte, 2013, pp. 33 sq.

44. Présentation du film The Ferryman sur le site Internet de sa maison de production : http:// www.lardux.com/article706

45. Emily Eliza Scott, «Artists' Platforms for New Ecologies », Third Text, vol. 27, $\mathrm{n}^{\circ} 1,2013$ : www.thirdtext.org/artists-platforms-for-new-ecologies-arc

46. Voir en ligne : www.worldofmatter.net

47. Cf. Ursula Biemann, Peter Mörtenböck et Helge Mooshammer, «From Supply Lines to Resource Ecologies », Third Text, vol. 27, n¹, 2013, pp. 76-94

48. Ibid., p. 77 (nous traduisons). 
49. Gentiane Bélanger, "World of Matter, ou la pensée complexe des territoires ", Espace: Art actuel, $n^{\circ} 110,2015$, p. 53.

50. Cf. Xavier Ribas, Nitrate, Barcelone, Museu d'Art Contemporani de Barcelona (MACBA), 2014.

51. Hélène Guenin (dir.), Sublime. Les tremblements du monde, Metz, Centre Pompidou-Metz, 2016, p. 126.

52. Voir notamment sur le photographe Burtynsky : Jennifer Peeples, « Toxic Sublime: Imaging Contaminated Landscapes », Environmental Communication, vol. 5, n²4, 2011, pp. 373-392.

53. Unknown Fields Division est un collectif artistique dirigé par Liam Young et Kate Davies qui s'est notamment donné pour tâche de chroniquer les arrières-soutes de la mondialisation, les paysages de l'extractivisme, de la manufacture mondiale, de la logistique conteneurisée...

54. Il y a d'autres exemples récents de démarches comparables, comme celle d'Anne Fisher qui a reçu le prix COAL 2017 avec son projet Rising from its Ashes, la création d'émaux utilisant des plantes hyperaccumulatrices, qui extraient puis stockent les métaux lourds des substrats pollués d'une ancienne mine de zinc des Cévennes.

55. John Dewey, Logique. La théorie de l'enquête, traduit par Gérard Deledalle, Paris, Presses universitaires de France, 1967, p. 300.

56. Cf. Dario Cimorelli (dir.), Le Prix Marcel Duchamp 2017. Maja Bajevic, Charlotte Moth, Vittorio Santoro, Joana Hadjithomas et Khalil Joreige, Cinisello Balsamo, Silvana, 2017.

57. Pour l'exposition, les artistes conservent seize carottes au total (sur les 350 prélevées) provenant de quatre sites : Louvre et Collège de France pour Paris, Athènes et Beyrouth.

58. Dewey, Logique, p. 297.

59. Pour Bruno Latour, le problème des Modernes est d'avoir façonné des " amalgames » entre des modes d'existence distincts. cf. Enquête sur les Modes d'existence. Une anthropologie des modernes, Paris, La Découverte, 2012, pp. 106 sq.

60. Laurent Rigoulet, "Sur la mer agitée de Leviathan avec Lucien Castaing-Taylor et Verena Paravel ", Télérama, 2013 : www.telerama.fr/cinema/sur-la-mer-agitee-avec-lucien-castaingtaylor-et-verena-paravel-realisateurs-de-leviathan,101522.php

61. Cyril Neyrat, "Blood of the Fish, Beauty of the Monster", in Bruno Latour (dir.), Reset Modernity!, Cambridge, MIT Press, 2016, p. 165 (nous traduisons).

\section{RÉSUMÉS}

Cet article se propose d'explorer des stratégies artistiques contemporaines qui se trouvent sinon en consonance avec l'hypothèse d'un Anthropocène, du moins en étroit voisinage avec les incidences stimulantes de cette notion sur les sciences-humaines. Se gardant toutefois d'aplatir la création contemporaine sur une seule acception philosophique de l'Anthropocène, l'auteur propose un panorama d'une avant-garde artistique qui s'attache moins aux questions de «représentation» de la nature qu'aux démarches d'enquête qu'adoptent les artistes afin d'intensifier leurs expériences d'un milieu naturel dévasté par la Modernité. L'expression « habiter le crassier » signifie en l'occurrence qu'il appartient à l'art de développer une fonction résidentielle à l'intérieur des «ruines du capitalisme» (Anna Tsing), en prenant appui sur une « économie générale » (Georges Bataille) de la nature anthropisée.

The aim of this article is to explore contemporary art strategies which are, maybe not strictly consistent with an Anthropocene hypothesis, but at least close to the stimulating implications of 
this notion for the social sciences. The author of the article, who is unwilling to foist contemporary works on a single philosophical understanding of the Anthropocene, provides an overview of an artistic avant-garde which is less and less interested in how to "represent" nature and more and more in the empirical approaches favored by artists in order to get more intense experience of natural environments that have been devastated by Modernity. "Living in the slag heap" implies that it is the responsibility of art to nurture a residential function in the midst of the "ruins of capitalism" (Anna Tsing), using a "general economy" (Georges Bataille) of humanmade nature.

\section{AUTEUR}

\section{MATTHIEU DUPERREX}

Docteur en arts plastiques, Matthieu Duperrex est chercheur associé au LLA-Créatis (EA 4152), Université de Toulouse-Jean Jaurès. Artiste-auteur, co-fondateur et directeur artistique du collectif « Urbain, trop urbain » (www.urbain-trop-urbain.fr), ses travaux procèdent d'enquêtes de terrain sur des milieux anthropisés et croisent littérature, sciences humaines et arts visuels ou numériques. Publications : Shanghai Nø City Guide (Toulouse, Urbain, trop urbain, 2012), Micromegapolis, lorsqu'une ville rencontre Gaïa (Toulouse, Urbain, trop urbain, 2013), Périphérique intérieur (Marseille, Wildproject, 2014), Voyages en sol incertain, enquête dans les deltas du Rhône et du Mississippi (Marseille, Wildproject, 2019).

Matthieu Duperrex has a PhD in visual arts and is a member of the LLA-Créatis research center at Université Toulouse Jean Jaurès. He is also an artist and the co-founder of an organization dedicated to the visual arts called "Urbain trop urbain". His research is predicated on field work and revolves around the implications of the Anthropocene from the perspective of literature, the human sciences and digital and visual arts. His works include Shanghai Nø City Guide (Toulouse, Urbain, trop urbain, 2012), Micromegapolis, lorsqu'une ville rencontre Gaïa (Toulouse, Urbain, trop urbain, 2013), Périphérique intérieur (Marseille, Wildproject, 2014), Voyages en sol incertain, enquête dans les deltas du Rhône et du Mississippi (Marseille, Wildproject, 2019). 